\title{
La contienda por la educación
}

Carlos Ornelas, México, FCE, 2018, 437 p.

\section{Philippe Rabaté}

\section{CpenEdition}

\section{Journals}

Édition électronique

URL : https://journals.openedition.org/ries/8525

DOI : $10.4000 /$ ries.8525

ISSN : 2261-4265

Éditeur

France Education international

Édition imprimée

Date de publication : 1 septembre 2019

Pagination : 23-24

ISBN : 978-2-85420-624-1

ISSN : $1254-4590$

Référence électronique

Philippe Rabaté, «La contienda por la educación », Revue internationale d'éducation de Sèvres [En ligne], 81 | septembre 2019, mis en ligne le 01 septembre 2019, consulté le 14 octobre 2021. URL : http:// journals.openedition.org/ries/8525; DOI : https://doi.org/10.4000/ries.8525

Ce document a été généré automatiquement le 14 octobre 2021.

(c) Tous droits réservés 


\title{
La contienda por la educación
}

\author{
Carlos Ornelas, México, FCE, 2018, 437 p.
}

Philippe Rabaté

\section{RÉFÉRENCE}

La contienda por la educación, Carlos Ornelas, México, FCE, 2018, 437 p.

1 Dans son dernier ouvrage, Carlos Ornelas, l'un des meilleurs spécialistes des politiques éducatives latino-américaines, nous offre une analyse très fine du cas mexicain à partir d'une perspective globale qui donne à son étude une profondeur et une validité saisissantes. Le point de départ et d'aboutissement de ce volume est la réforme de l'éducation lancée par le président Enrique Peña Nieto (2012-2018) dès le début de son mandat. Considérée comme l'une des mesures phares de sa présidence, celle-ci a d'abord fait l'objet d'un vote des deux chambres (décembre 2012) avant de devenir loi constitutionnelle (février 2013), et son contenu impliquait une véritable rupture dans les pratiques existantes, avec la mise en place d'un service national d'évaluation et de gestion des écoles ainsi qu'avec le développement d'une plus grande autonomie des établissements scolaires.

2 Afin de rendre compte de ce vaste chantier, encore inachevé au moment où l'auteur achève la rédaction de son étude à la fin de l'année 2017, Carlos Ornelas propose à son lecteur d'élargir son champ de vision en recourant à des concepts qu'il a pu forger tout au long de sa carrière professorale et scientifique. Aussi débute-t-il son ouvrage par une instructive typologie des réformes éducatives, qu'il tend à définir à partir de critères judicieux (objectifs, formes, acteurs impliqués, durée, motivation des parties concernées, etc.). Après ce chapitre liminaire, il se penche successivement sur trois modèles qui lui semblent constituer les principaux acteurs de cette lutte qui agite le champ éducatif mexicain. Une première dynamique mondiale s'exprime dans une conception néolibérale de l'éducation qui fait de celle-ci un véritable marché où tendent à s'équilibrer l'offre et la demande. Cette considération économique de l'enseignement est notamment présente dans des organismes intergouvernementaux 
comme l'OCDE, à laquelle le Mexique appartient depuis le virage libéral pris sous la présidence de Carlos Salinas de Gortari (1988-1994).

3 Toutefois, le pays fait preuve de résistance face aux dogmes libéraux ou globaux, principalement en raison de la puissance du syndicat le plus important historiquement, le Syndicat national des travailleurs de l'éducation (SNTE), qui constitue un véritable État dans l'État. Ornelas consacre des réflexions passionnantes à la montée hégémonique de cet organisme, qui est parvenu à dominer le processus de nomination des nouveaux maîtres, en privilégiant notamment les charges héréditaires (un enfant peut ainsi obtenir un poste d'enseignant parce que l'un de ses parents en détenait un sans qu'il y ait le moindre processus de contrôle) et, quand la transmission générationnelle n'est pas possible, en insti- tuant une vente au plus offrant. Au-delà de la corruption évidente que de telles pratiques peuvent engendrer, le SNTE tend à dominer l'ensemble du système éducatif en développant une forme de néocorporatisme que ne parvient pas à vaincre la secrétaire à l'éducation publique (SEP), intégrée au ministère de l'éducation. Ornelas évoque en dernier lieu un modèle démocratique qui a de toute évidence sa préférence: l'éducation n'y est plus une marchandise ni un objet soumis aux appétits corporatistes mais un droit inaliénable et universel pour chaque enfant.

4 Une fois posé ce théâtre conceptuel, l'auteur se lance, à partir du chapitre VI, dans une étude fine et rigoureuse de la réforme du président Peña Nieto. La lutte qu'elle prétend instaurer contre des pratiques néo-corporatistes au nom de principes hérités à la fois de l'approche néolibérale et démocratique donne lieu à des tensions très fortes avec le SNTE, qui tente de bloquer par tous les moyens les bouleversements liés à l'instauration de la réforme. Cette oscillation entre modèle global et démocratique tend par ailleurs à brouiller l'action du gouvernement, sans compter la résistance des maitres, de plus en plus grande. Ornelas étudie avec brio l'usure de la crédibilité et du pouvoir présidentiels, notamment grevés par la disparition à Iguala, dans le sud du pays, de 43 étudiants d'école normale en septembre 2014 alors même que ceux-ci se rendaient à une manifestation contre l'action gouvernementale. L'onde de choc qui a secoué toute la société mexicaine n'a d'ailleurs toujours pas cessé et cette affaire tragique a suscité une défiance considérable à l'égard du président en exercice.

5 Toujours est-il que le combat pour dominer l'éducation s'est durci sur fond de mutation progressive du rôle de l'État, devenu à la fois évaluateur et capable de développer une nouvelle gestion publique. Dans ce contexte, si le néocorporatisme est encore la force dominante dans le pays, il se voit miné et attaqué par les tendances démocratiques et néolibérales (chapitre VIII). Carlos Ornelas se concentre ensuite (chapitre IX) sur ce qui constitue, selon lui, la cible principale de la réforme, à savoir les enseignants à travers le dispositif de la loi du service professionnel pour les enseignants, qui offre un véritable référentiel pour la carrière des enseignants (heures, évaluation, poursuite de carrière, etc.), inexistant jusqu'alors. Son regard s'arrête ensuite sur une mise en perspective de la réforme à partir d'une vision globale et non limitée au Mexique, en abordant successivement le point de vue des partisans d'une culture mondiale face aux réussites et résistances de la réforme, puis la vision des opposants à l'intervention d'organismes internationaux d'inspiration libérale comme l'OCDE. Ce second type d'approche oublie, selon l'auteur, l'importance des acteurs nationaux et la très forte singularité historique du Mexique. 
6 Le livre se clôt sur un épilogue où Carlos Ornelas livre une appréciation pondérée et suggestive de la réforme Peña Nieto par-delà tous les éléments polémiques dont nous avons brièvement fait état. Malgré ses insuffisances et défauts, la réforme semble aller en partie dans le sens d'une approche démocratique de l'éducation, ce dont se réjouit l'auteur.

7 La contienda por la educación est un ouvrage remarquable qui sait alterner étude précise de l'histoire mexicaine et considérations séduisantes sur les réformes éducatives. La réforme n'a toutefois pas survécu à l'alternance politique, et l'un des premiers gestes du Président López Obrador a été d'abroger la loi désirée et conçue par son prédécesseur, signe - si besoin en était - de la difficile continuité de la politique éducative mexicaine.

\section{AUTEUR}

\section{PHILIPPE RABATÉ}

Philippe Rabaté, maître de conférences, dirige le département d'espagnol de l'Université ParisOuest-Nanterre-La Défense. Il est membre associé du comité de rédaction de la Revue internationale de Sèvres. Courriel : philippe.rabate@u-paris10.fr 